\title{
Rheological and Dynamic Mechanical Properties of Abutilon Natural Straw and Polylactic Acid Biocomposites
}

\author{
Hua Wang $\mathbb{D}^{1}{ }^{1}$ Hafeezullah Memon $\mathbb{D}^{2,3}$ Elwathig A. M. Hassan, ${ }^{2,4,5}$ Tienah H. H. Elagib, \\ Fadl Elmoula A. A. Hassan $\left(\mathbb{D},{ }^{5}\right.$ and Muhuo Yu ${ }^{2,4}{ }^{2,4}$ \\ ${ }^{1}$ College of Textiles, Donghua University, 2999 North Renmin Road, Shanghai 201620, China \\ ${ }^{2}$ Donghua University Center for Civil Aviation Composites, Donghua University, 2999 North Renmin Road, Shanghai 201620, China \\ ${ }^{3}$ Key Laboratory of Textile Science \& Technology, Ministry of Education, College of Textiles, Donghua University, 2999 North \\ Renmin Road, Shanghai 201620, China \\ ${ }^{4}$ State Key Laboratory for Modification of Chemical Fibers and Polymer Materials, Donghua University, Shanghai 200051, China \\ ${ }^{5}$ Industries Engineering and Technology, University of Gezira, Wad Madani 21111, Sudan
}

Correspondence should be addressed to Fadl Elmoula A. A. Hassan; kadabas_20@yahoo.com and Muhuo Yu; yumuhuo@dhu.edu.cn

Received 22 May 2019; Revised 28 August 2019; Accepted 26 September 2019; Published 7 December 2019

Academic Editor: Matthias Schnabelrauch

Copyright (C) 2019 Hua Wang et al. This is an open access article distributed under the Creative Commons Attribution License, which permits unrestricted use, distribution, and reproduction in any medium, provided the original work is properly cited.

\begin{abstract}
Recently, natural fibers have become attractive materials to engineers, scientists, and researchers as an alternative reinforcement for biocomposites. In this study, polylactic acid/abutilon natural straw biocomposites with various abutilon straw weight fractions were prepared by melt blending. The differential scanning calorimetric (DSC) results showed a significant influence of the abutilon straw on the melting behavior of PLA, even at the low abutilon straw contents. The dynamic mechanical analysis demonstrated that the storage modulus, as well as tan delta of the biocomposites, increased when the abutilon straw content increases, which indicates better interaction between abutilon natural straw and PLA. The incorporation of abutilon straw into biocomposites provided favorable changes in rheology related to the matrix. SEM observation revealed good dispersion of the abutilon straw in PLA.
\end{abstract}

\section{Introduction}

In recent years, the requirement for environmentally friendly materials is increasing to reduce the consumption of fossil fuels and prevent global environmental concern $[1,2]$. Natural reinforced composites are used widely replacing synthetic reinforced polymers, especially for industrial application such as the automotive, construction, and packaging industries $[3,4]$. Investigations have also been carried out to produce composites from natural fiber using different types of matrices, such as polypropylene [5], polyethylene [6], epoxy [7], and polyhydroxybutyrate [8], which is capable of satisfying many engineering applications in terms of energy, strength, and absorption. PLA is a synthetic aliphatic polyester produced from renewable agriculture products such as sugarcane, sugar beet maize, and cassava. PLA is well known as a biodegradable and biocompatible polymer [9]. However, the brittleness, relatively high cost, and melt viscosity for further processing limited its further development for broad application $[10,11]$. Several studies were carried out to investigate the possibility of PLA-reinforced natural fiber to improve biocomposite properties. It has been reported that different types of fiber such as hemp, flax, jute, bamboo, and kenaf as well as sisal were used, because of the abundant availability, low price, low density, and excellent mechanical properties [12-15]. Most of these studies revealed that some properties like tensile and flexural strength were improved. Liu et al. [16] stated that the sizing of basalt fiber significantly increases tensile, impact, and strength bending of PLA. Also, the study conducted by Huda et al. [17] examined the PLA/recycle cellulose composite property by using extrusion and injection molding and stated that the filler (up to 30\%) improved the rigidity without hindering the degree of crystallinity and thermal stability. 
Nevertheless, the growing interest of natural composite materials and the applications of these composites are limited to the nonstructural field because of disadvantages such as poor compatibility with the matrix and relatively high moisture absorption $[18,19]$. It has been known that natural fibers contain an amount of cellulose, hemicellulose, lignin, and pectin which make natural fibers susceptible to moisture absorption [20]. Many physical and chemical surface modifications have been employed to boost the compatibility between thermoplastic matrix and natural fibers to achieve high-performance natural fiber-reinforced biocomposites $[21,22]$. Jandas et al. [23] investigated the treatment of banana fibers/PLA biocomposite fabricated by melt mixing and compression molding, and the result showed that the morphology analysis indicated a very significant improvement of surface wetting and adhesion properties.

In this study, the abutilon straw was selected as the reinforcement for PLA composites. Abutilon straws generated from agriculture residue considered as renewable resources are widely available in Sudan. These are being burned to eliminate deforestation and have never been utilized in building a new material application. Burning these materials further raises the issues related to global warming. The effect of added polylactic acid on morphology, thermal, dynamic mechanical, and rheological properties was investigated to take full advantage of abutilon straw as a new building material.

\section{Materials and Methods}

2.1. Materials. The polylactic acid (PLA, 4032D-grade) in pellet form used as a matrix resin was purchased from Unic Technology (Suzhou) Co., Ltd., China. Sodium hydroxide $(\mathrm{NaOH})$ and acetone $(\geq 99.5 \%)$ were supplied by Shanghai Yunli Economic and Trading Co., Ltd., China.

2.2. Straw Collection and Treatment. The abutilon straws were collected from the University of Gezira farm, then washed with water to remove dust and impurities from the surface. Abutilon straws were crushed to a powder form (average diameter was $45 \mu \mathrm{m}$ ), then further washed with acetone to remove impurities and treated with $\mathrm{NaOH}$. Finally, the treated straws were dried in an oven at $75^{\circ} \mathrm{C}$ for $72 \mathrm{hr}$.

2.3. Composite Preparation. The pellets of the PLA matrix and abutilon straw powder were initially dried in a vacuum oven at a temperature of $70^{\circ} \mathrm{C}$ for $24 \mathrm{~h}$ to remove the water before processing through the extruder. Biocomposites with abutilon straw content from 1, 3, and 5\% were mixed in a container using a high-speed mixer. The final mixtures were fed into a corotating twin-screw at $100 \mathrm{rpm}$ at $180-190^{\circ} \mathrm{C}$ to obtain the granules. The injection molding was used to form blended samples into the standard test. The temperature of the injection molding was $180-190^{\circ} \mathrm{C}$ at the pressure of $1 \mathrm{MPa}$ and the mold temperature about $40^{\circ} \mathrm{C}$.

2.4. Characterizations. The functional groups of PLA, straw, and PLA/straw were identified by Fourier transform infrared (FTIR) spectrometer (Nicolet 8700, USA) using powderpressed $\mathrm{KBr}$ disks in the wavenumber range from $4000 \mathrm{~cm}^{-1}$ to $400 \mathrm{~cm}^{-1}$. Thermogravimetric analysis (TGA) was carried out under $\mathrm{N}_{2}$ atmosphere using a TGA Q5000 IR (TA Instruments-Waters LLC, New Castle, DE, USA) and a sample weight of 3-5 grams with a heating rate of $15^{\circ} \mathrm{C} / \mathrm{min}$ from $30^{\circ} \mathrm{C}$ to $450^{\circ} \mathrm{C}$. Thermal characterization of the biocomposites was performed with DSC Q20 (TA InstrumentsWaters LLC) (sample weight: 5-10 mg, temp: 30-250 ${ }^{\circ} \mathrm{C}$ ).

Dynamic mechanical analysis (DMA) was carried out using a DMA Q800 (TA Instruments-Waters). The dimension of the rectangular specimens was $60 \times 7.5 \times 2.5 \mathrm{~mm}$ tested by dual cantilever method. The measurements were determined at a frequency of $1 \mathrm{~Hz}$ and a strain rate of $0.1 \%$, at temperature ranges from $40^{\circ} \mathrm{C}$ to $120^{\circ} \mathrm{C}$ with a heating rate of $4^{\circ} \mathrm{C} / \mathrm{min}$. Rheological properties of the blends and pure resins were measured using ARES-RFS (TA Instruments-Waters). The frequency range was set at $0.1 \sim 100 \mathrm{~Hz}$. Before the measurement, the samples were prepared using injection molding at $180^{\circ} \mathrm{C}$. The fracture morphology of composites was observed using a scanning electron microscope (SEM) (HITACHI S-300N, Japan) with an acceleration voltage of $(30 \mathrm{kV})$.

\section{Results and Discussion}

3.1. Fourier Transformed Infrared Spectroscopy. FTIR spectroscopy is a useful technique to determine the functional groups of abutilon straw and abutilon straw/PLA biocomposites. As shown in Figure 1, the broad peaks at $3421 \mathrm{~cm}^{-1}$ and $602 \mathrm{~cm}^{-1}$ are associated with $-\mathrm{OH}$ functional groups in the abutilon straw sample. The absorbance peaks around $1049 \mathrm{~cm}^{-1}$ were attributed to the $\mathrm{C}-\mathrm{O}$ group, the peak at $2925 \mathrm{~cm}^{-1}$ is related to the $\mathrm{C}-\mathrm{H}$ group stretching in an aromatic methyl group of cellulose and hemicellulose, and the peak at $1629 \mathrm{~cm}^{-1}$ is associated with the $\mathrm{C}=\mathrm{C}$ group attributed to aromatic skeletal vibration of lignin. The peak at $1737 \mathrm{~cm}^{-1}$ corresponds to the $\mathrm{C}=\mathrm{O}$ group. The $\mathrm{OH}$ group peaks disappeared for the abutilon straw/PLA biocomposites, indicating that the PLA well-wrapped abutilon straw, which is attributed to good interaction adhesion.

3.2. DSC Analysis. The melt-crystallization DSC curves of pure PLA and PLA/abutilon straw biocomposites at a cooling rate of $10^{\circ} \mathrm{C} / \mathrm{min}$ are shown in Figure 2. It was observed that the peak related to glass transition temperature (Tg) in PLA completely disappeared in DSC thermographs with the addition of abutilon straw. The disappearance of the Tg suggests that the amorphous phase of PLA was predominantly hydrolyzed and removed [24]. Moreover, the addition of abutilon straw into the PLA matrix has not significantly increased the melting temperature (Tm) value for PLA/abutilon straw biocomposites. The slight improvement in melting temperature might be due to the strong interaction adhesion between the abutilon straw and PLA polymer. Furthermore, the result suggested that the abutilon straw not only improves crystallization but also hinders the motion of the PLA matrix chain in the melting process.

3.3. Thermogravimetric Analysis. As shown in Figure 3, the pure PLA underwent a one-stage degradation from $251^{\circ} \mathrm{C}$ 


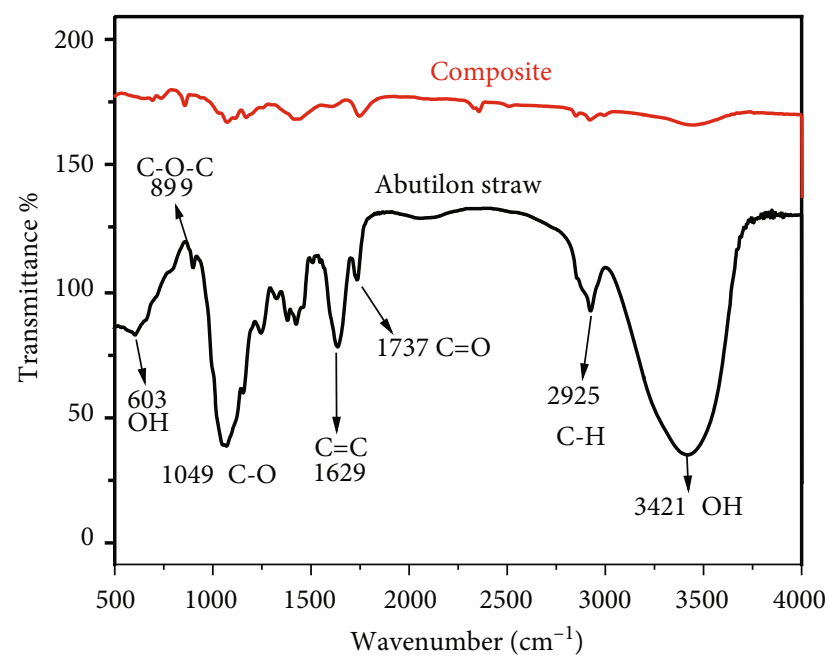

FIGURE 1: FTIR spectra of abutilon straw and PLA/abutilon straw biocomposites.

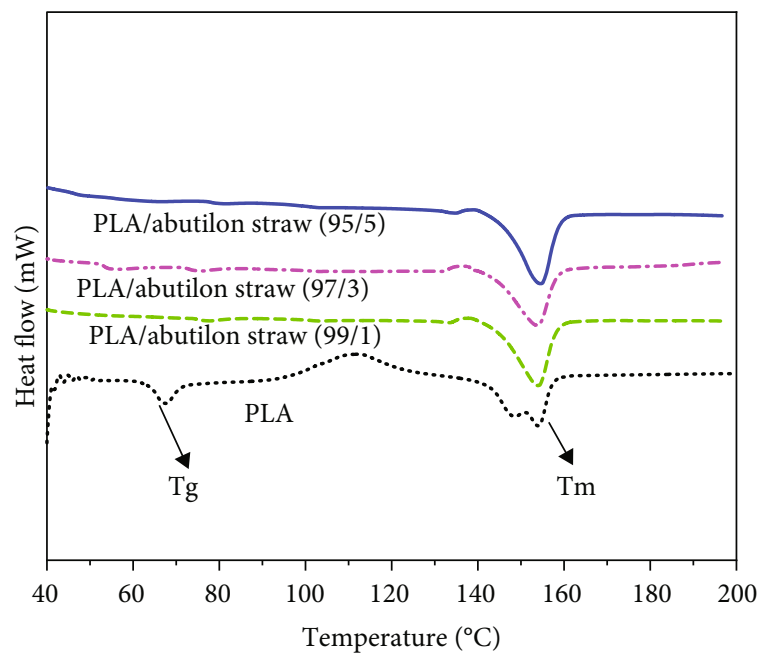

Figure 2: DSC curves of PLA and PLA/abutilon straw biocomposites.

and experienced the highest degradation rate at around $272^{\circ} \mathrm{C}$. It produced more than $20 \%$ char residue at $\sim 500^{\circ} \mathrm{C}$. As for the PLA/abutilon straw, the thermal degradation process started earlier compared with that of pure PLA and further decreased with increasing abutilon straw content that can be attributed to lower degradation temperature of abutilon straw.

3.4. Dynamic Mechanical Analysis. Dynamic mechanical behaviors of pure PLA and PLA/abutilon straw biocomposites were studied by using DMA. It was observed from Figure 4(a) that storage modulus of PLA increased with the incorporation of abutilon straw. The better stress transfer explains the improvement of the storage modulus within abutilon straw-reinforced composites [25]. These results showed that the pure PLA had a modulus of $1475 \mathrm{MPa}$ at $45^{\circ} \mathrm{C}$. The storage modulus increased to $1585 \mathrm{MPa}$,

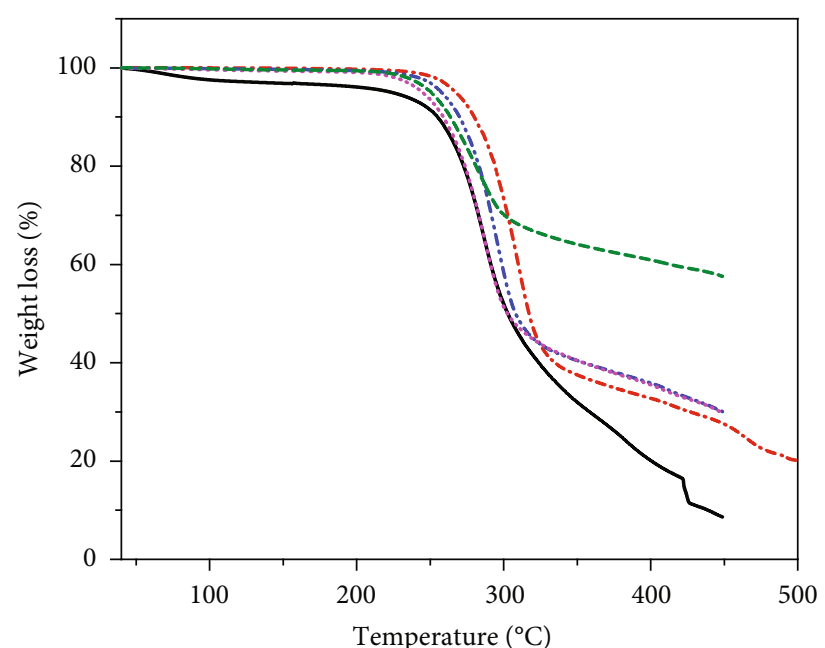

$\begin{array}{lll}\text { - Abutilon straw } & \text {--- } & \text { PLA/abutilon straw (97/3) } \\ \text {..-- PLA } & \text { PL... } & \text { PLA/abutilon straw (95/5) } \\ \text {..... PLA/abutilon straw (99/1) } & \end{array}$

Figure 3: TGA curves of PLA and PLA/abutilon straw biocomposites.

$1782 \mathrm{MPa}$, and $1800 \mathrm{MPa}$ for PLA/abutilon straw (99/1), PLA/abutilon straw (97/3), and PLA/abutilon straw (95/5), respectively. The improvement of the storage modulus is due to better interaction between the treated abutilon straw and the PLA matrix. $\tan \delta$ can provide information about how good material can absorb energy. Figure 4(b) shows the $\tan \delta$ of the pure PLA and PLA/abutilon straw biocomposites as a function of temperature. The $\tan \delta$ height of biocomposites was reduced compared with pure or neat PLA and was further reduced with increasing abutilon straw content. The phenomenon indicates that the abutilon straw is highly compatible with the PLA matrix, which is attributed to less energy dissipation and greater restricted mobility at the interface [26].

3.5. Rheological Properties. Figure 5 shows the storage modulus $\left(G^{\prime}\right)$ and loss modulus $\left(G^{\prime \prime}\right)$ of neat or pure PLA and PLA/abutilon straw biocomposites as a function of frequency at $180^{\circ} \mathrm{C}$. The rheological properties of the PLA/abutilon straw biocomposites showed lower values to that of PLA. Processability of the composites is influenced by the addition of abutilon straw to the polymer melts. The storage modulus values of PLA/abutilon straw (97/3) except (95/5) biocomposites are higher than that of the pure matrix at all frequency ranges. This behavior can be explained by the fact that plenty of abutilon straw particles restrict deformation, which showed a reinforcing effect in PLA. The differences in storage modulus of the PLA/abutilon straw samples could be attributed to their differences in particle loading. The relation between frequency and $\tan \operatorname{delta}\left(\tan \delta=\mathrm{G}^{\prime \prime} / \mathrm{G}^{\prime}\right)$ was analyzed to see the behavior of PLA/abutilon straw biocomposites to lose energy to molecular rearrangements and internal friction. In this composite system, as shown in Figure $5(\mathrm{c}), \tan$ delta $(\tan \delta)$ decreased with the addition of abutilon straw, which is mainly due to the existence of 


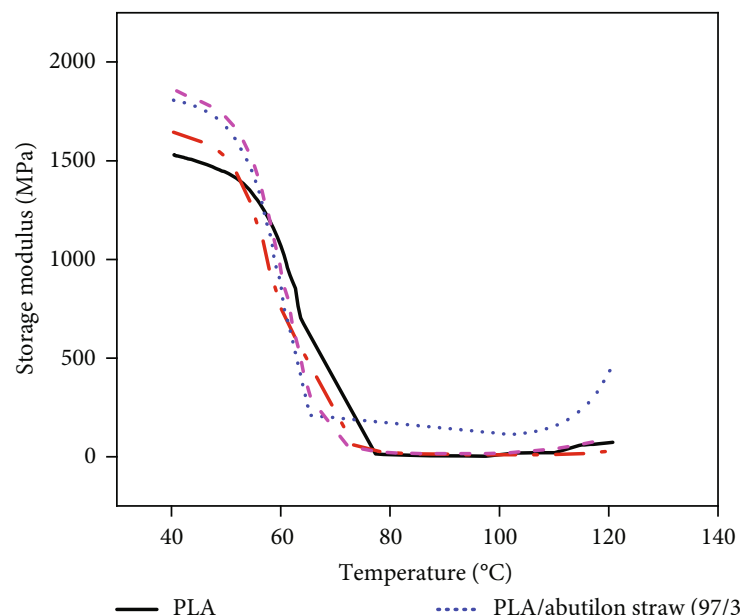

.-. PLA/abutilon straw (99/1) - - - PLA/abutilon straw (95/5)

(a)

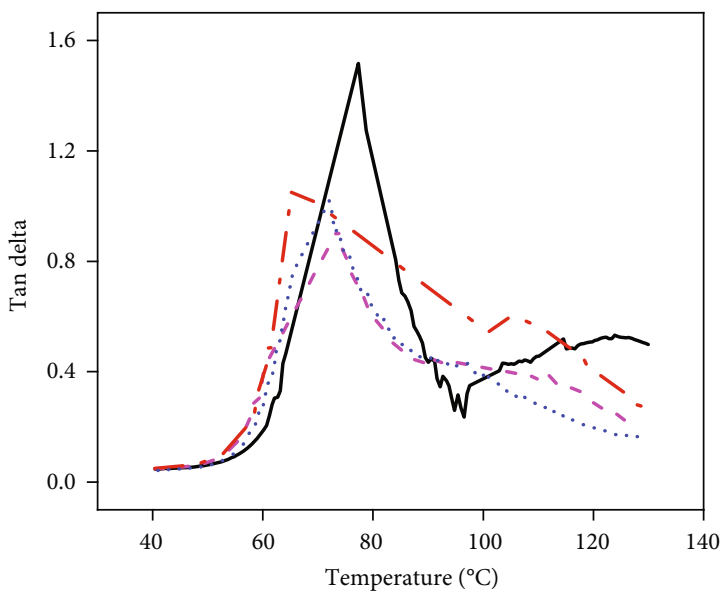

- PLA $\quad$..... PLA/abutilon straw (97/3) -... PLA/abutilon straw (99/1) --.- PLA/abutilon straw (95/5)

(b)

FIGURE 4: Dynamic mechanical analysis: (a) storage modulus; (b) tan $\delta$ of PLA and PLA/abutilon straw biocomposites.

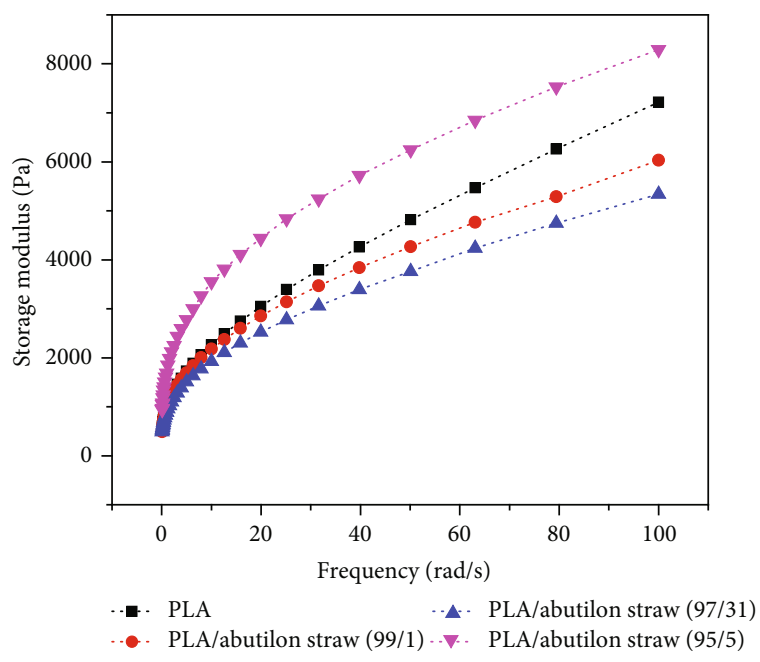

(a)

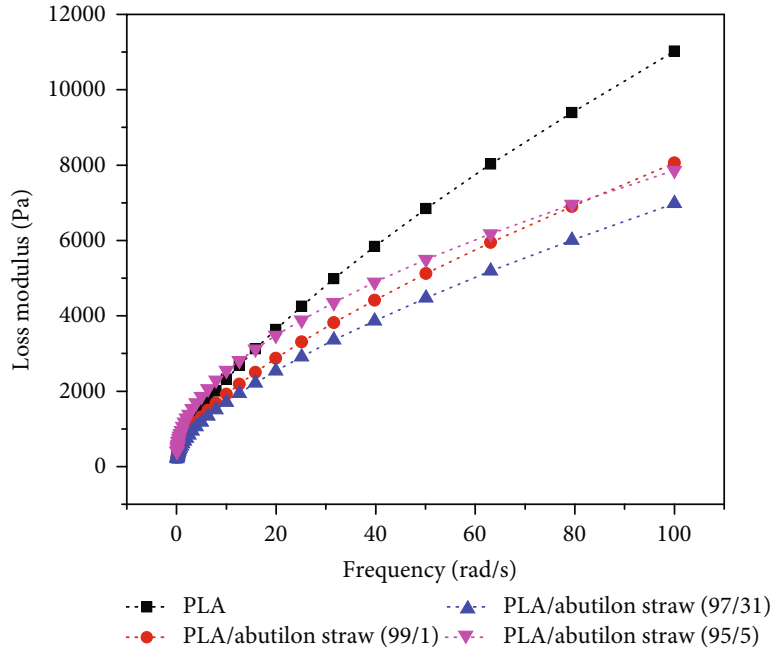

(b)

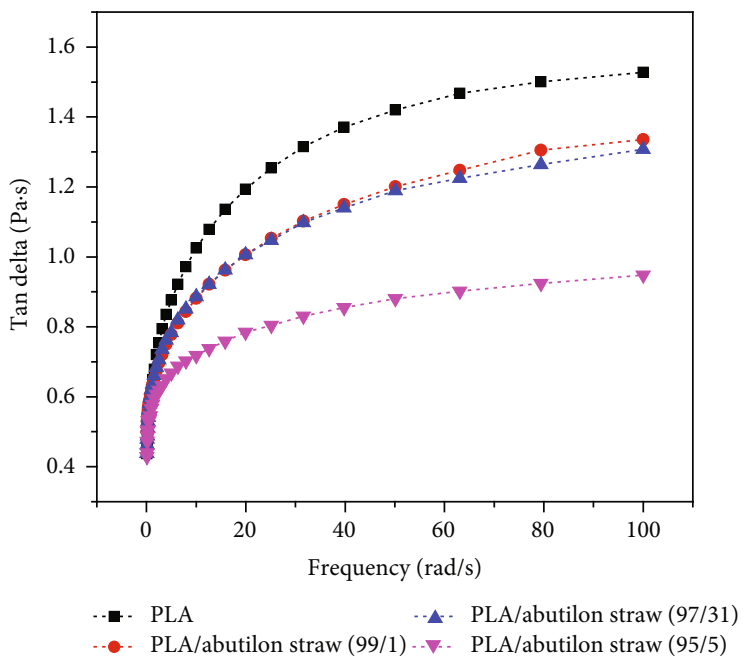

(c)

FIGURE 5: Rheological properties: (a) storage modulus; (b) loss modulus; (c) tan delta of PLA and PLA/abutilon straw biocomposites. 

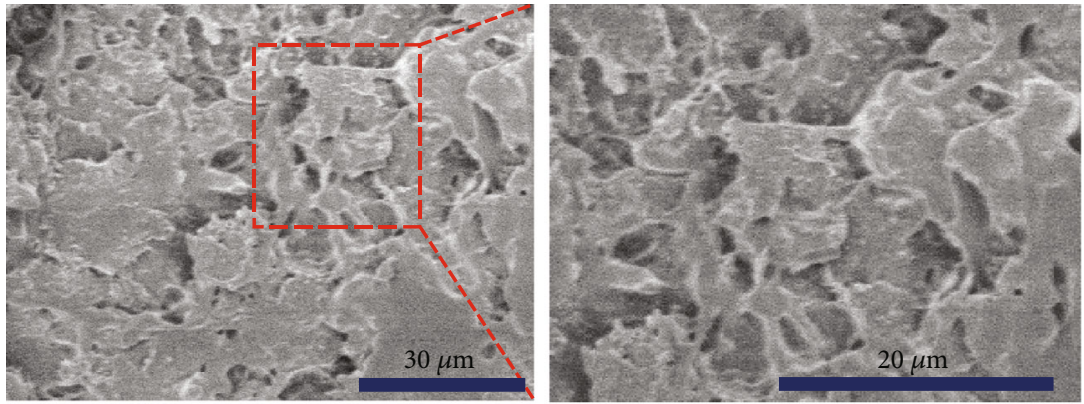

(a)
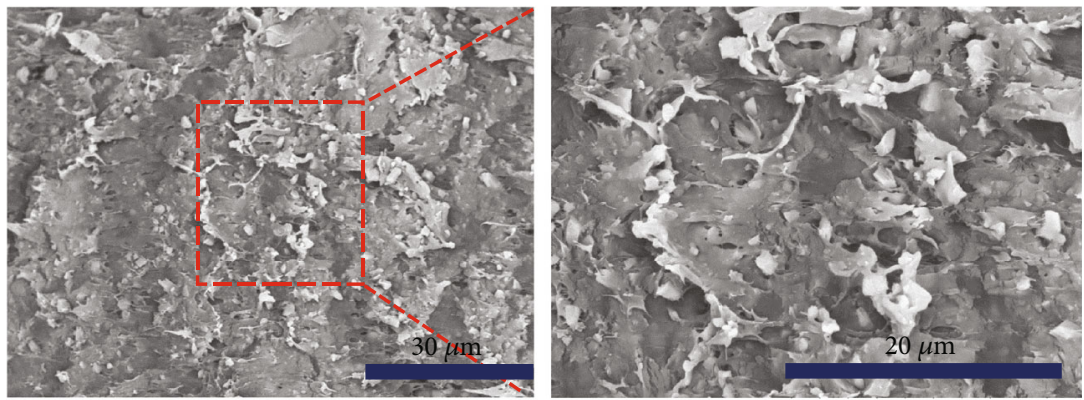

(b)
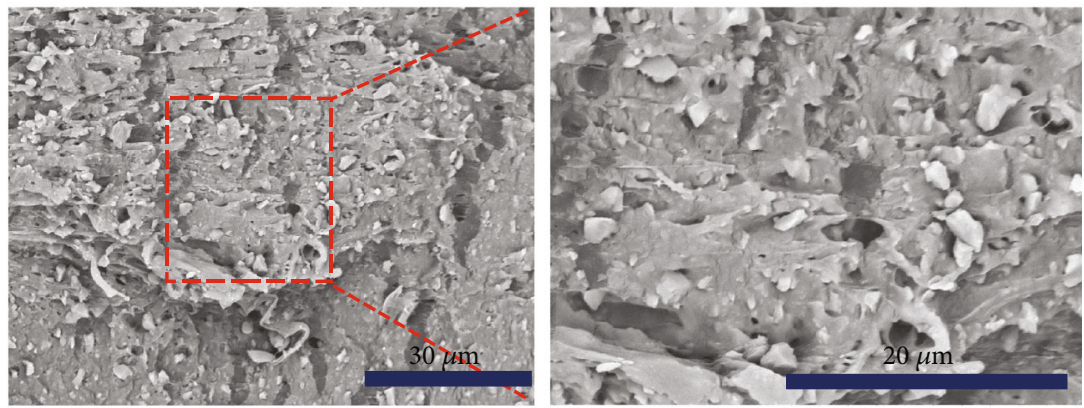

(c)
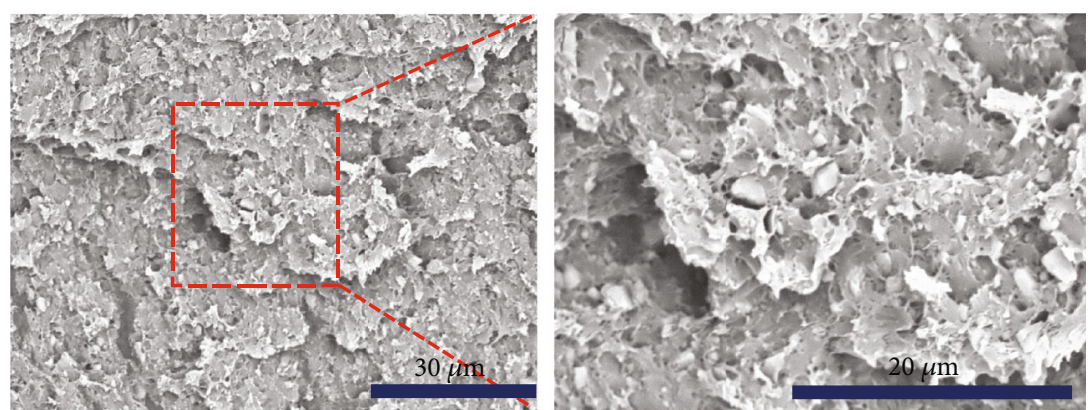

(d)

Figure 6: Composite morphology analysis by SEM images: (a) pure PLA, (b) PLA/abutilon straw (99/1), (c) PLA/abutilon straw (97/3), and (d) PLA/abutilon straw (95/5) biocomposites with a length scale of $20 \mu \mathrm{m}$ and $30 \mu \mathrm{m}$.

effective interaction between abutilon straw and the PLA matrix. Therefore, the viscoelastic energy dissipation in the composites was limited [27]. The melt rheological tests for the aged PLA and PLA composites also showed the evidence of differences in the flowing behavior depending on the abutilon straw content.
3.6. Composite Morphology Analysis. Well dispersion of straw and the interaction between straw and the PLA matrix played a vital role in determining the mechanical performance of final composites. The fractured surfaces of PLA composites were scanned by SEM. From Figure 6(a), it can be seen that pure PLA showed a relatively smooth fractured surface. 


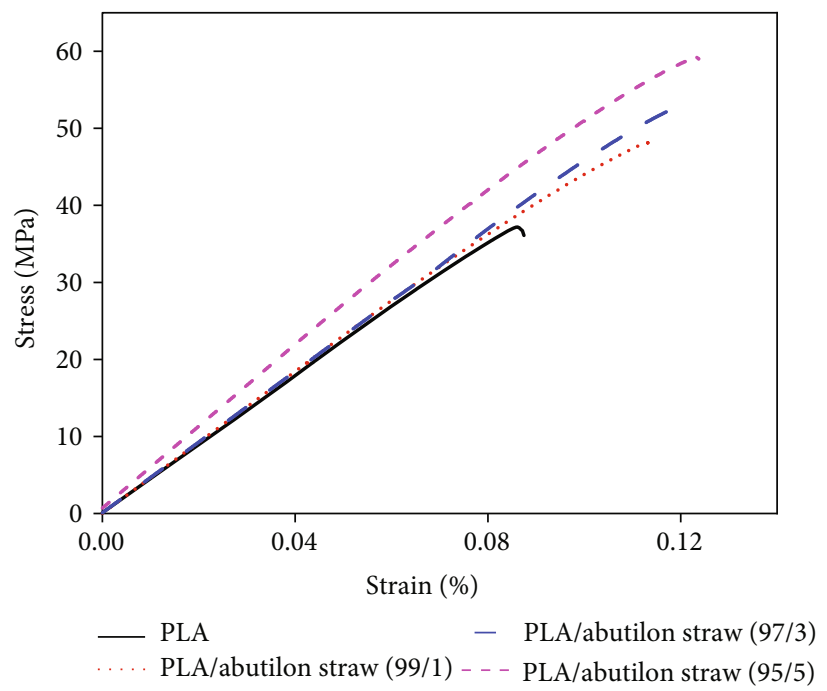

(a)

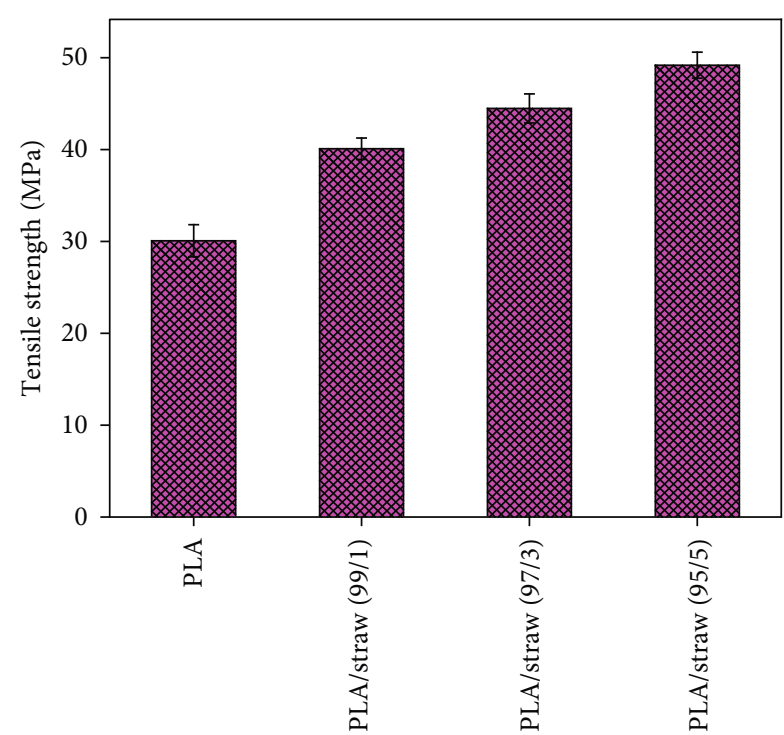

(b)

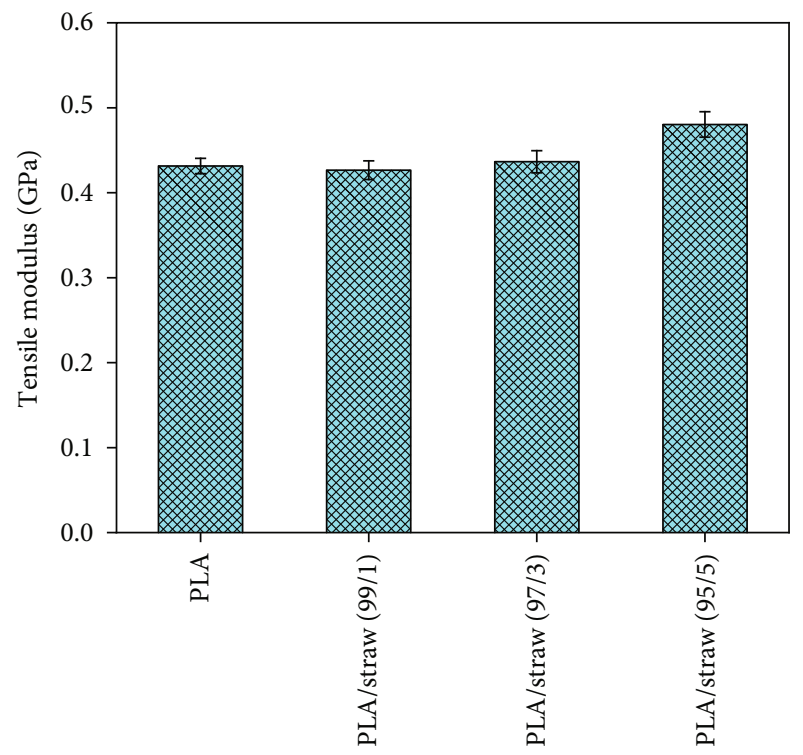

(c)

Figure 7: (a) Stress-strain curve, (b) tensile strength, and (c) tensile modulus.

Figure 6(b)-6(d) shows a good abutilon straw dispersion in the PLA matrix and the abutilon straw particles were not agglomerated. Moreover, when the abutilon straw loading increased, the composite fracture surface was rugged and rough. Furthermore, the abutilon straw surface appears to be covered by a layer of the PLA polymer. This result indicates stronger adhesion between abutilon straw and the PLA matrix, as well as homogeneous dispersion of abutilon straw within the PLA matrix, was achieved, which allowed efficient stress transfer between abutilon straw and the polymer matrix.

3.7. Mechanical Properties of the Composite. The effect of the incorporation of abutilon straw on the mechanical performance of the composites was also studied. The stress-strain curve of neat PLA and PLA/abutilon straw composites are shown in Figure 7(a). The figure clearly shows that the tensile stress and elongation to break are higher for the composites compared to pure PLA. The addition of abutilon straw particles significantly increased the tensile strength and modulus of PLA, as shown in Figures 7(b) and 7(c), further enhanced with increasing abutilon straw ratio. For instance, the tensile strength was increased from $37.18 \pm 1.2 \mathrm{MPa}$ to $53.8 \pm 2.4$ $\mathrm{MPa}$ and the modulus from $0.429 \pm 0.1$ to $0.483 \pm 0.15 \mathrm{GPa}$ for the neat PLA to the composites with $5 \mathrm{wt} . \%$ straw content, respectively. The result indicated that the PLA could be successfully reinforced by straw.

\section{Conclusions}

Herein, we studied the PLA/abutilon natural straw biocomposites with $1 \%, 3 \%$, and $5 \%$ abutilon straws. It was found that an increased content of abutilon straw in the PLA resulted in a decrease in the decomposition temperature, 
indicating reduced thermal stability of the PLA. The tensile strength and modulus of the PLA/abutilon straw biocomposites increased with the increasing of abutilon straw content. Thus, the dispersion of straw in PLA, as well as the interaction between PLA and the straw, was improved. This result was supported by SEM analyses which proved good dispersion of the abutilon straw in the PLA. The DMA results revealed that the straw-reinforced biocomposites have higher storage modulus resulting in significantly improved stiffness and storage modulus, which further increased with increasing straw content. The direct relation between straw content and tan delta also proved the better interaction between abutilon natural straw and PLA. However, the rheological properties decreased with the abutilon straw addition, the most significant effect seen again with the further increasing straw content. This study might be further extended to see the effect of temperature on the rheological properties.

\section{Data Availability}

All the data is available on request by emailing to fadlalmawala@uofg.edu.sd.

\section{Conflicts of Interest}

The authors declare that there is no conflict of interest regarding the publication of this paper.

\section{Acknowledgments}

All laboratory staff of the College of Textiles, Donghua University, China, is gratefully acknowledged.

\section{References}

[1] D. B. Dittenber and H. V. S. Gangarao, "Critical review of recent publications on use of natural composites in infrastructure," Composites Part A Applied Science \& Manufacturing, vol. 43, no. 8, pp. 1419-1429, 2012.

[2] H. Memon, A. Khatri, N. Ali, and S. Memon, "Dyeing recipe optimization for eco-friendly dyeing and mechanical property analysis of eco-friendly dyed cotton fabric: better fixation, strength, and color yield by biodegradable salts," Journal of Natural Fibers, vol. 13, pp. 749-758, 2016.

[3] S. A. Hinchcliffe, K. M. Hess, and W. V. Srubar III, "Experimental and theoretical investigation of prestressed natural fiber- reinforced polylactic acid (PLA) composite materials," Composites Part B: Engineering, vol. 95, pp. 346-354, 2016.

[4] H. Kyutoku, N. Maeda, H. Sakamoto, H. Nishimura, and K. Yamada, "Effect of surface treatment of cellulose fiber (CF) on durability of PLA/CF bio-composites," Carbohydrate Polymers, vol. 203, pp. 95-102, 2019.

[5] I. Angelov, S. Wiedmer, M. Evstatiev, K. Friedrich, and G. Mennig, "Pultrusion of a flax/polypropylene yarn," Composites Part A: Applied Science and Manufacturing, vol. 38, no. 5, pp. 1431-1438, 2007.

[6] F. Z. Arrakhiz, M. El Achaby, A. C. Kakou et al., "Mechanical properties of high density polyethylene reinforced with chemically modified coir fibers: impact of chemical treatments," Materials \& Design, vol. 37, pp. 379-383, 2012.
[7] E. Sarikaya, H. Çallioğlu, and H. Demirel, "Production of epoxy composites reinforced by different natural fibers and their mechanical properties," Composites Part B: Engineering, vol. 167, pp. 461-466, 2019.

[8] A. da Silva Moura, R. Demori, R. M. Leão, C. L. Crescente Frankenberg, and R. M. Campomanes Santana, "The influence of the coconut fiber treated as reinforcement in PHB (polyhydroxybutyrate) composites," Materials Today Communications, vol. 18, pp. 191-198, 2019.

[9] E. Fortunati, D. Puglia, J. M. Kenny, M. Minhaz-Ul Haque, and M. Pracella, "Effect of ethylene-co-vinyl acetateglycidylmethacrylate and cellulose microfibers on the thermal, rheological and biodegradation properties of poly(lactic acid) based systems," Polymer Degradation and Stability, vol. 98, no. 12, pp. 2742-2751, 2013.

[10] R.-y. Chen, W. Zou, H.-c. Zhang et al., "Thermal behavior, dynamic mechanical properties and rheological properties of poly(butylene succinate) composites filled with nanometer calcium carbonate," Polymer Testing, vol. 42, pp. 160-167, 2015.

[11] A. Hassan, H. Balakrishnan, and A. Akbari, "Polylactic acid based blends, composites and nanocomposites," in Advances in natural polymers, pp. 361-396, Springer, 2013.

[12] R. Dungani, H. A. Khalil, I. Sumardi et al., "Non-wood renewable materials: properties improvement and its application," in Biomass and Bioenergy, pp. 1-29, Springer, 2014.

[13] K. Makhijani, R. Kumar, and S. K. Sharma, "Biodegradability of blended polymers: a comparison of various properties," Critical Reviews in Environmental Science and Technology, vol. 45, no. 16, pp. 1801-1825, 2015.

[14] N. Saba, M. T. Paridah, and M. Jawaid, "Mechanical properties of kenaf fibre reinforced polymer composite: a review," Construction and Building Materials, vol. 76, pp. 87-96, 2015.

[15] H. Wang, H. Memon, E. A. M. Hassan, M. S. Miah, and M. A. Ali, "Effect of jute fiber modification on mechanical properties of jute fiber composite," Materials, vol. 12, no. 8, p. 1226, 2019.

[16] T. Liu, F. Yu, X. Yu, X. Zhao, A. Lu, and J. Wang, "Basalt fiber reinforced and elastomer toughened polylactide composites: mechanical properties, rheology, crystallization, and morphology," Journal of Applied Polymer Science, vol. 125, no. 2, pp. 1292-1301, 2012.

[17] M. S. Huda, A. K. Mohanty, L. T. Drzal, E. Schut, and M. Misra, “"Green” composites from recycled cellulose and poly (lactic acid): physico-mechanical and morphological properties evaluation," Journal of Materials Science, vol. 40, no. 16, pp. 4221-4229, 2005.

[18] C. A. Fuentes, G. Brughmans, L. Q. N. Tran, C. DupontGillain, I. Verpoest, and A. W. Van Vuure, "Mechanical behaviour and practical adhesion at a bamboo composite interface: physical adhesion and mechanical interlocking," Composites Science and Technology, vol. 109, pp. 40-47, 2015.

[19] F. K. Liew, S. Hamdan, M. R. Rahman, and M. Rusop, “Thermomechanical properties of jute/bamboo cellulose composite and its hybrid composites: the effects of treatment and fiber loading," Advances in Materials Science and Engineering, vol. 2017, Article ID 8630749, 10 pages, 2017.

[20] A. K. Mohanty, M. Misra, and L. T. Drzal, "Surface modifications of natural fibers and performance of the resulting biocomposites: an overview," Composite Interfaces, vol. 8, no. 5, pp. 313-343, 2001.

[21] M. M. Kabir, H. Wang, K. T. Lau, and F. Cardona, "Chemical treatments on plant-based natural fibre reinforced polymer 
composites: an overview," Composites Part B: Engineering, vol. 43, no. 7, pp. 2883-2892, 2012.

[22] Y. Xie, C. A. S. Hill, Z. Xiao, H. Militz, and C. Mai, "Silane coupling agents used for natural fiber/polymer composites: a review," Composites Part A: Applied Science and Manufacturing, vol. 41, no. 7, pp. 806-819, 2010.

[23] P. J. Jandas, S. Mohanty, S. K. Nayak, and H. Srivastava, "Effect of surface treatments of banana fiber on mechanical, thermal, and biodegradability properties of PLA/banana fiber biocomposites," Polymer Composites, vol. 32, no. 11, pp. 1689-1700, 2011.

[24] M. R. N. Fazita, K. Jayaraman, D. Bhattacharyya, M. S. Hossain, M. K. M. Haafiz, and H. P. S. Abdul, "Disposal options of bamboo fabric-reinforced poly(lactic) acid composites for sustainable packaging: biodegradability and recyclability," Polymers, vol. 7, p. 1465, 2015.

[25] L. Zhang, Z. Li, Y.-T. Pan et al., "Polydopamine induced natural fiber surface functionalization: a way towards flame retardancy of flax/poly(lactic acid) biocomposites," Composites Part B: Engineering, vol. 154, pp. 56-63, 2018.

[26] S. Jia, D. Yu, Y. Zhu, Z. Wang, L. Chen, and L. Fu, "Morphology, crystallization and thermal behaviors of PLA-based composites: wonderful effects of hybrid GO/PEG via dynamic impregnating," Polymers, vol. 9, no. 12, p. 528, 2017.

[27] A. O. Ogah, J. N. Afiukwa, and A. A. Nduji, "Characterization and comparison of rheological properties of agro fiber filled high-density polyethylene biocomposites," Open Journal of Polymer Chemistry, vol. 04, no. 01, pp. 12-19, 2014. 


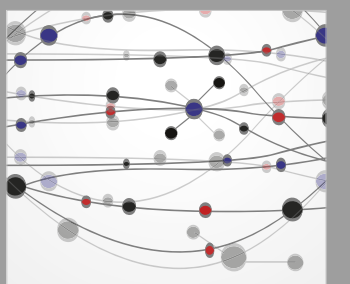

The Scientific World Journal
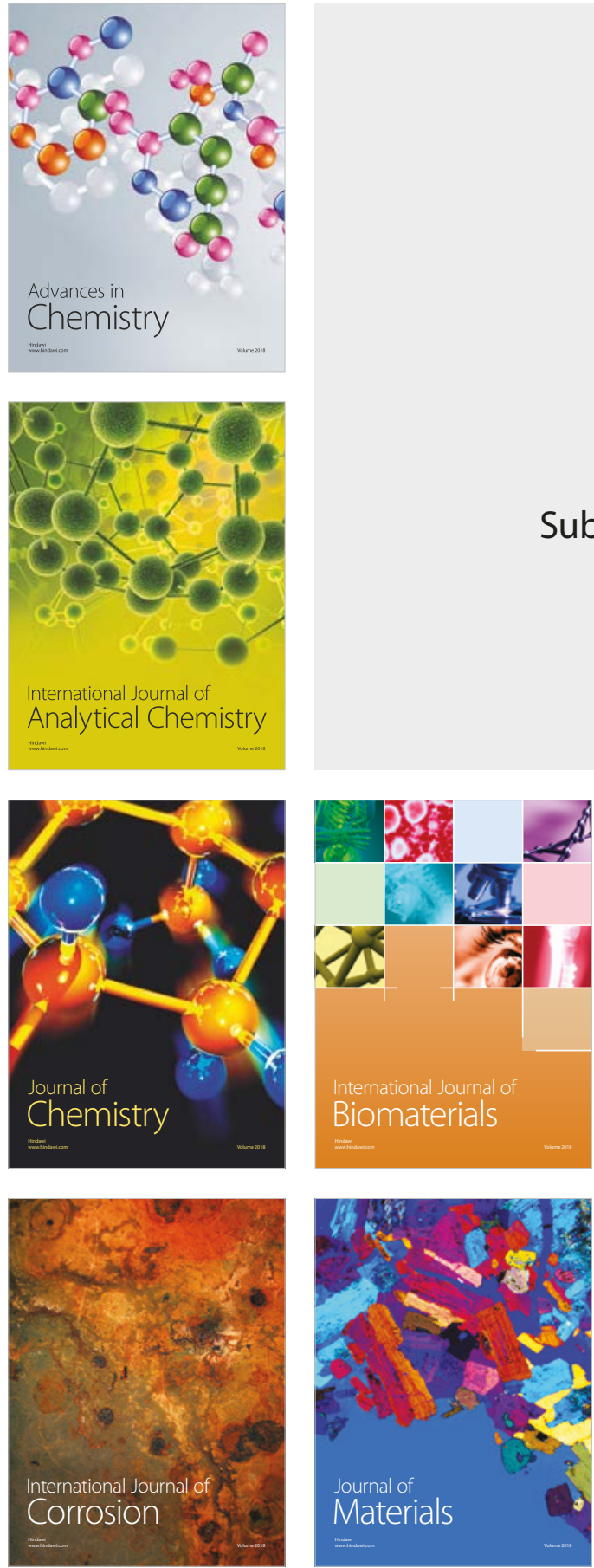

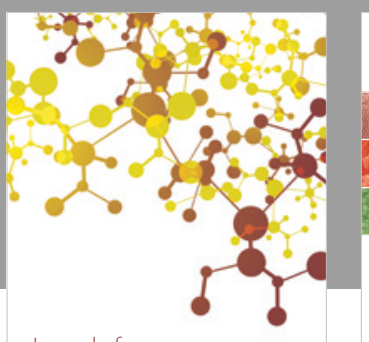

Journal of

Applied Chemistry
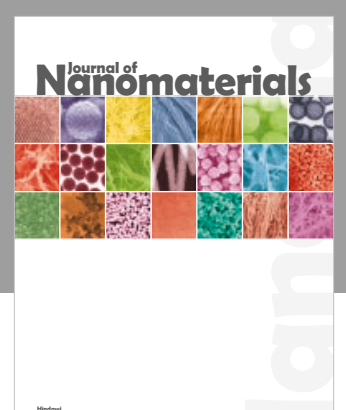

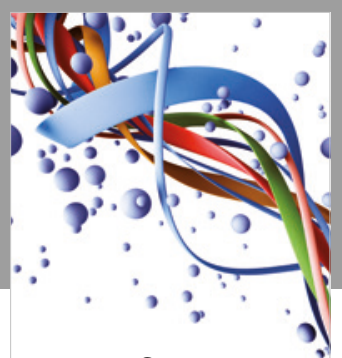

Scientifica

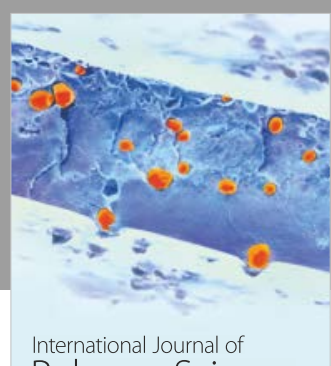

Polymer Science

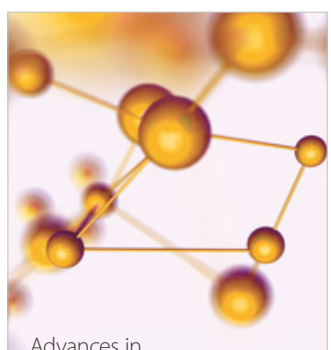

Physical Chemistry
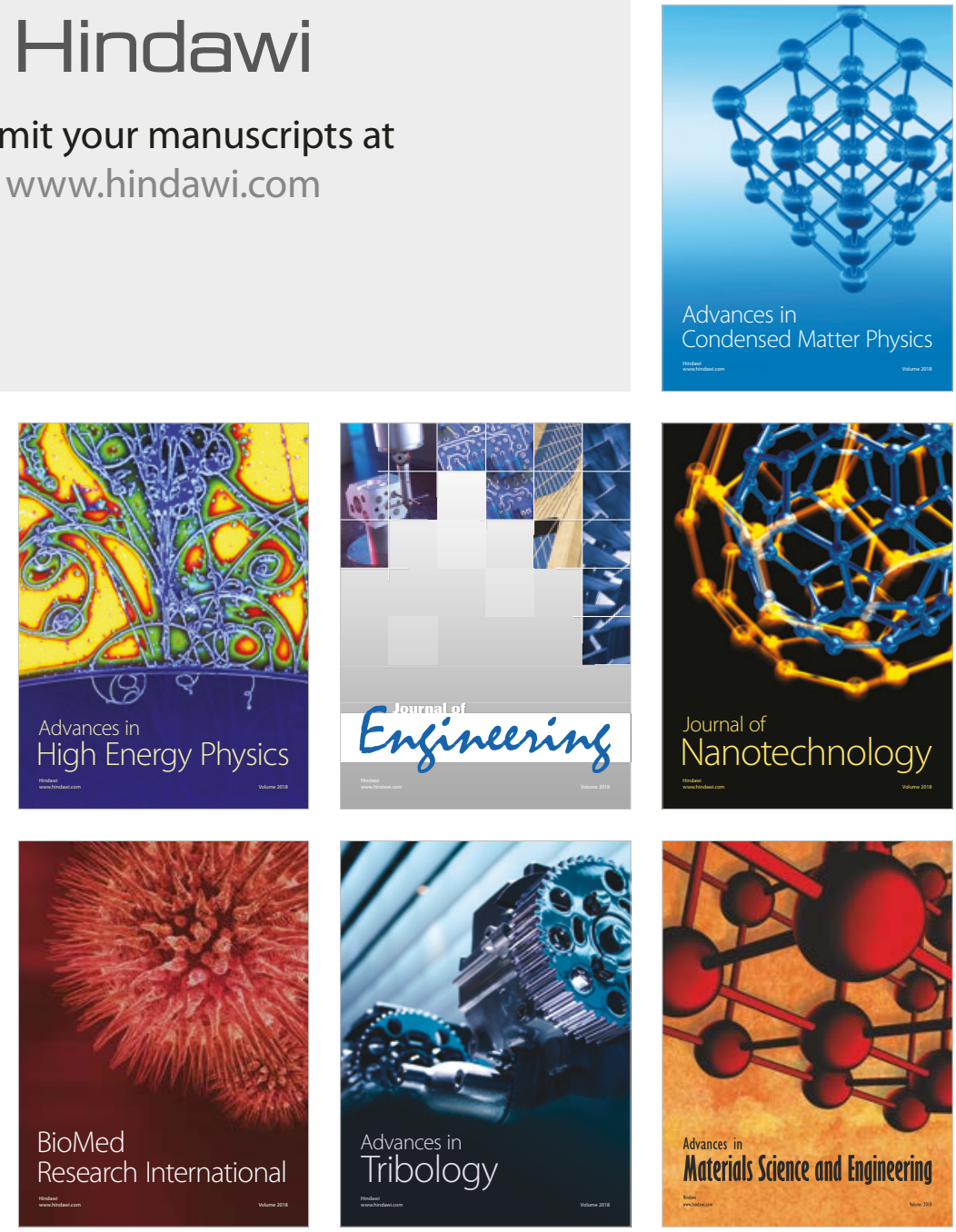